$\chi^{2}=11.08$, d.f. $\left.=2, P<0.005\right)$ but not male schizophrenics $\left(n=8089, \chi^{2}=2.93\right.$, d.f. $=2$, NS $)$. The estimated peak for female schizophrenics, and for both sexes taken together, was early May.

The existence of significant seasonality in part supports the findings of our England and Wales study. This study is, however, weak, in that we were not, at that time, able to obtain the data on first admissions for mania in Scotland, and could not compare the seasonal variation of female schizophrenics with that of manics. Nevertheless, the gender difference in season of admissions in schizophrenia persisted in a second large data set. However, the estimated peak of admissions in this study precedes by two months that of our English data, which showed a July peak of admissions for female schizophrenics. Although the reason for this discrepancy is unclear, some geographical or climatic factor(s) may play a role. The delay in the peak of admissions in England and Wales compared with Scotland is at odds with the idea that increasing warmth or lengthened daylight is responsible for the increase in admissions.

We thank Dr S. K. Cole, Scottish Health Service, Common Services Agency, Edinburgh, for providing us with data.

EDWARDS, J. H. (1961) The recognition and estimation of cyclic trends. Annals of Human Genetics, 25, 83-86.

TAKel, N., O'Callaghan, E., Sham, P., et al (1992) Seasonality of admissions in the psychoses: effect of diagnosis, sex, and age at onset. British Journal of Psychiatry. 161, 506-511.

R. M. MURRAY
Department of Psychological Medicine
Institute of Psychiatry \& King's College Hospital
De Crespigny Park
London SES 8AF

\section{Estimating premorbid IQ in schizophrenia}

SIR: Crawford et al (Journal, July 1992, 161, 69-74) reported comparisons between controls and schizophrenic samples on the Wechsler Adult Intelligence Scale (WAIS; Wechsler, 1958) scores and National Adult Reading Test (NART; Nelson, 1982) scores; community resident and long-stay hospital patients had significantly lower verbal and performance WAIS scores than controls, whereas only long-stay patients had poorer NART scores than controls. They discussed the efficacy of using the NART to estimate premorbid IQ but no conclusions were reached as to whether the low scores in the longstay sample were a true indication of low premorbid IQ or whether "NART performance was impaired by onset of disease". We wish to draw attention to a methodological point regarding the study design and to a general problem in using a reading test such as the NART in disorders beginning in early adult life.

In order to draw conclusions on the effects of schizophrenia on test results, cases and controls should have been comparable, other than for the presence of schizophrenia. The absence of long-term in-patients in the control group for long-stay patients may have introduced bias; poor intellectual functioning may make life in long-stay care more likely, regardless of schizophrenia. Also, the effects of neuroleptic drugs, present only in the cases, may have attenuated test performance. If, as Crawford et al suggest, NART score and, to a lesser extent, WAIS verbal skills are relatively resistant to the ravages of neuropathology, compared with performance skills, they may also be resistant to the deleterious effects of neuroleptics. Drug dosages and test scores could be examined for such dose-response relationships.

Use of the NART as a measure of premorbid IQ has become increasingly common in studies of cognitive ageing and dementia, and has been extended to other conditions (Crawford, 1989). However, its applicability to schizophrenia and other disorders of early adulthood raises difficulties because the considered explanations of poor performance are not comprehensive. Knowledge of the acquisition of intellectual skills in the normal population forms a useful foundation for hypotheses concerning the disruptive effects of pathology. The view that intellectual functioning reaches a peak in the teenage years has been supplanted by the less rigid notion of significant advancement later, possibly much later, in life and of different age gradients for different skills. The empirical evidence is sparse concerning the acquisition of reading skills but suggests a substantial improvement for the general population through the late teenage years and early adulthood, perhaps continuing into middle life (Rodgers, 1986). One might predict similar gains for the NART. That statistically significant age gradients have not been reported (Crawford, 1989) may be due to relatively small samples and the inextricable confounding of age and cohort effects in cross-sectional study designs.

If an appreciable gain in reading skills occurs in the general population over the age range representing the maximum risk period for incidence of schizophrenia (or other disorders), patients may show a slower rate or cessation of improvement as illness impinges on their normal activities. Under this model, a relative deficit in NART performance reflects neither erosion of previously attained 
capabilities nor low premorbid IQ. The degree to which expected gains are not realised is likely to be a function of both severity and age at onset of the disease. Deficits could result from either an impairment of the capacity to benefit from learning circumstances, or a reduction in the occurrence of those circumstances, such as through institutionalisation, or both. Strictly speaking, a reduced rate of skill acquisition could be said to come under the umbrella of impairment by disease onset but there are important empirical and theoretical distinctions between this model and notions such as 'resistance' or 'holding-up' of performance. There are also differences between the two formulations when considering useful indicators of premorbid intelligence. Clearly, the contemporary skills assessed must be resistant to pathology but should also reach an early developmental plateau in the general population.

The importance of considering the age at onset of disorders under study is highlighted by the implications of Crawford's conclusion that in psychiatric/psychological research, schizophrenic cases should be matched to controls on the basis of premorbid IQ. If, as many believe, some schizophrenic psychoses are an age-dependent manifestation of a pathological process occurring during early brain development (Jones \& Murray, 1991) such a general strategy would leave schizophrenia researchers with neither the bath water nor the baby, although it might be useful in disorders with unequivocal late onset. At present, reading tests such as the NART provide a more accurate assessment of premorbid intellectual function in schizophrenia than can be obtained by many alternative tests. However, we believe that it is unwise to ignore the difficulties associated with its use, either for individuals or for groups of patients, and premature to apply the term premorbid IQ to adjusted NART scores as has occurred in recent publications (Nelson et al, 1990; Frith et al, 1991).

CRAWFord, J. R. (1989) Estimation of premorbid intelligence: a review of recent developments. In Developments in Clinical and Experimental Neuropsychology (eds J. R. Crawford \& D. M. Parker), pp. 55-74. New York: Plenum Press.

Frith, C. D., Leary, J., Cahill, C., et al (1991) Disabilities and circumstances of schizophrenic patients - a follow-up study. IV. Performance on psychological tests: demographic and clinical correlates of the results of these tests. British Journal of Psychiatry, 159 (suppl. 13), 26-29.

Jones, P. B. \& MURRAY, R. M. (1991) The genetics of schizophrenia is the genetics of neurodevelopment. British Journal of Psychiatry, 158, 615-623.

NeLSON, H. E. (1982) National Adult Reading Test (NART) for the Assessment of Premorbid Intelligence in Patients with Dementia: Test Manual. Windsor: NFER Nelson.

- Pantelis, C., Carruthers, K., el al (1990) Cognitive functioning and symptomatology in chronic schizophrenia. Psychological Medicine, 20, 357-365.
RODGERS, B. (1986) Change in the reading attainment of adults: a longitudinal study. British Journal of Developmental Psychology, 4, 1-17.

WeChSLER, D. (1958) The Measurement and Appraisal of Adult Intelligence. 4th edn. Baltimore: Williams \& Wilkins.

Department of Psychological Medicine

Peter Jones

Institute of Psychiatry

De Crespigny Park

London SE5 8AF

NH \& MRC Social Psychiatry Unit

BRYAN RODGERS

Canberra

Australia

\section{Catatonia and not neuroleptic malignant syndrome}

SIR: The simplest explanation for the case reported by White (Journal, October 1992, 161, 558-560) is not that the patient experienced separate episodes of catatonia and neuroleptic malignant syndrome (NMS) as claimed, but that the patient had repeated episodes of catatonia only and never experienced NMS. The case description suggests this in three separate ways: (a) the sufficiency of endogenous catatonia and neuroleptic-induced dystonia to explain all findings, (b) the extremely beneficial response to parenteral benzodiazepines, and (c) the much lower exposure to neuroleptics than in cases of NMS.

This 33-year-old woman received only $25 \mathrm{mg}$ /day of chlorpromazine. In comparison, the mean dose of neuroleptic associated with NMS is equivalent to over $1000 \mathrm{mg} /$ day of chlorpromazine, and causative doses as low as $300 \mathrm{mg} /$ day are unusual (Pope et al, 1986; Shalev et al, 1989). We can attribute the withdrawal, fearfulness, agitation, stupor, autonomic instability, leukocythemia, and elevated muscle tone and enzyme levels before the first admission to catatonic psychosis. The observed dramatic clinical response to intravenous diazepam distinctively demonstrates that the patient suffered from catatonia and not NMS, because only the former (Greenfield et al, 1987) is dramatically sensitive to parenteral benzodiazepines. Indeed, response to parenteral lorazepam or intravenous amobarbital or diazepam should distinguish catatonia from NMS, although false negatives are possible.

The presentation of the second episode was neuroleptic-free, and the patient was therefore clearly purely catatonic. While the fever, tachypnoea, tachycardia, diaphoresis, creatine phosphokinase (CPK) elevation, and muscular rigidity that followed the injection of haloperidol $(10 \mathrm{mg})$ appears to be drug-related, it also required the presence of catatonia, as proven by the immediate resolution 